\title{
A wavelet analysis for the X-ray absorption spectra of molecules
}

\author{
T. J. Penfold, ${ }^{1,2,3, a)}$ I. Tavernelli, ${ }^{2}$ C. J. Milne,${ }^{3}$ M. Reinhard,${ }^{1}$ A. El Nahhas, ${ }^{1, b)}$ \\ R. Abela, ${ }^{3}$ U. Rothlisberger, ${ }^{2}$ and M. Chergui ${ }^{1}$ \\ ${ }^{1}$ Ecole polytechnique Fédérale de Lausanne, Laboratoire de spectroscopie ultrarapide, ISIC, FSB-BSP, \\ CH-1015 Lausanne, Switzerland \\ ${ }^{2}$ Ecole polytechnique Fédérale de Lausanne, Laboratoire de chimie et biochimie computationnelles, ISIC, \\ FSB-BCH, CH-1015 Lausanne, Switzerland \\ ${ }^{3}$ SwissFEL, Paul Scherrer Inst, CH-5232 Villigen, Switzerland
}

(Received 16 October 2012; accepted 6 December 2012; published online 3 January 2013)

\begin{abstract}
We present a Wavelet transform analysis for the X-ray absorption spectra of molecules. In contrast to the traditionally used Fourier transform approach, this analysis yields a 2D correlation plot in both $R$ - and $k$-space. As a consequence, it is possible to distinguish between different scattering pathways at the same distance from the absorbing atom and between the contributions of single and multiple scattering events, making an unambiguous assignment of the fine structure oscillations for complex systems possible. We apply this to two previously studied transition metal complexes, namely iron hexacyanide in both its ferric and ferrous form, and a rhenium diimine complex, $\left[\operatorname{ReX}(\mathrm{CO})_{3}(\mathrm{bpy})\right]$, where $\mathrm{X}=\mathrm{Br}, \mathrm{Cl}$, or ethyl pyridine (Etpy). Our results demonstrate the potential advantages of using this approach and they highlight the importance of multiple scattering, and specifically the focusing phenomenon to the extended X-ray absorption fine structure (EXAFS) spectra of these complexes. We also shed light on the low sensitivity of the EXAFS spectrum to the Re-X scattering pathway. (C) 2013 American Institute of Physics. [http://dx.doi.org/10.1063/1.4772766]
\end{abstract}

\section{INTRODUCTION}

$\mathrm{X}$-ray absorption spectroscopy (XAS) is a powerful tool for the investigation of the local geometric and electronic structure of molecules in solution. ${ }^{1-5}$ However, the complex origin of these spectra means that theoretical simulations are critical to obtain a complete analysis and to this end, the development of multiple scattering (MS) approaches ${ }^{6-9}$ revolutionised the domain, making it possible to simulate the whole spectrum in a computationally efficient manner. At present practical limitations of the MS approach usually restrict such simulations to the use of spherical muffin-tin potentials. ${ }^{10}$ As a consequence, these calculations are most reliable when the photoelectron is not sensitive to the fine details of the atomic potential, i.e., in the extended X-ray absorption fine structure (EXAFS) region which is the most attractive for local structural analysis.

In this region a core electron is excited well above $(>50 \mathrm{eV})$ the ionisation threshold and the identity of the surrounding atoms and their distances from the absorbing atom are characterised by the backscattering amplitude, $F_{\gamma}(k, R)$, of the photoelectron wave. Usually the initial step in obtaining a qualitative description of the structure is achieved using a Fourier transform (FT) of the EXAFS signal ${ }^{11}$ which yields a pseudo-radial distribution. ${ }^{12}$ Here, the scattering pathways appear as a peak in the FT, with a resolution of

$$
\Delta R \geq \pi / 2 \Delta k .
$$

\footnotetext{
a)Electronic mail: thomas.penfold@epfl.ch.

b) Present address: Chemical Physics, Lund University, P.O. Box 124, SE-22100 Lund, Sweden.
}

This means, for example, that data collected with a $\Delta k=11 \AA^{-1}$ will have a resolution $\Delta R=0.14 \AA$, and therefore scattering pathways which differ by $\leq \Delta R$ will appear as one peak. For systems which contain many scattering paths of different atomic species, and/or single and multiple scattering pathways which contribute to the same region of $R$-space, an unambiguous assignment of the peaks can be difficult. This is because these overlapping scattering pathways will alter the appearance of the FT and give rise to peaks which derive from a combination of multiple pathways, rather than one specific pathway. ${ }^{13}$ This is particularly pertinent if the data is Fourier filtered, i.e., certain distances are extracted in $R$-space and then back transformed into $k$-space to simplify the analysis for systems with low symmetry. In such cases, assigning and fitting features as if they are composed of a single scattering (SS) pathway, when in reality they arise from a mixture of many scattering pathways or of MS will give erroneous results.

A full analysis is generally performed using a fit of the experimental spectrum ${ }^{14}$ by means of the EXAFS equation ${ }^{2,7}$ which is expressed as

$$
\chi(k)=\sum_{\gamma} \frac{N_{\gamma} S_{0}^{2} F_{\gamma}(k, R)}{k R_{\gamma}^{2}} e^{-2 R_{\gamma} / \lambda(k)} e^{-2 \sigma^{2} k^{2}} \sin \left(2 k R_{\gamma}+\phi_{\gamma}\right) .
$$

Here, $\gamma$ is the scattering path index with degeneracy $\mathbf{N}_{\gamma}$. The half-path distance and the squared Debye-Waller factor are represented by $R_{\gamma}$ and $\sigma^{2}$, respectively. $\lambda(k)$ is the energydependent mean free path and $S_{0}^{2}$ is the amplitude reduction factor which accounts for many-body effects. The term $\sin \left(2 k R_{\gamma}+\phi_{\gamma}\right)$ describes the phase of the scattered photoelectron and is key to the peak positions in the FT. Here, $\phi_{\gamma}$ 
is the phase shift of the final state, which is expressed as

$$
\phi_{\gamma}(k)=2 \phi_{\gamma}^{\text {absorber }}(k)+\phi_{\gamma}^{\text {scatterer }}(k),
$$

describing the changes to the phase associated with the increase in velocity of the photoelectron as it approaches the neighbouring nuclei. ${ }^{15}$ This can usually be approximated as a linear function, except when heavy atoms are involved in the scattering pathway. ${ }^{7,16}$

In implementing such a procedure it is important to remember that a meaningful analysis should comply with the Nyquist criterion, ${ }^{17}$ describing the minimum number of independent points compared to the number of fitting variables. The finite number of data point for an EXAFS spectrum means that it is usually only possible to include a few of the important scattering pathways of the system, and as in the case of the pseudo-radial distribution discussed above. For systems with many closely correlated scattering pathways this can make a complete description of the structure quite challenging.

To address these limitations, in the present work we apply an approach based on a Wavelet transform (WT) analysis. Here, the infinitely expanded periodic oscillations of the FT are replaced by a local function, a wavelet, which enables one to analyse the components in $k$ - and $R$ space, concurrently. This yields a 2D correlation plot in both coordinates (analogous to a time-frequency correlation plot) and will separate the contributions between different scattering pathways (to within $\mathrm{Z} \pm 1^{7}$ ) at the same distance from the absorbing atom and between the contributions of SS and MS events because they will display different $k$ dependencies. Despite its advantages, the WT analysis has not been implemented to study the EXAFS spectra of molecular systems, and it has only been used in a few occasions for solid state systems. ${ }^{18-21}$ Here, we apply it to study the EXAFS spectra of two transition metal complexes, namely iron hexacyanide in both its ferric and ferrous form, and a rhenium diimine complex, $\left[\operatorname{ReX}(\mathrm{CO})_{3}(\mathrm{bpy})\right]$, where $\mathrm{X}=\mathrm{Br}, \mathrm{Cl}$, or ethyl pyridine (Etpy). Our analysis shows that for the former we are able to extract details of the small structural changes between the ferric and ferrous complexes and highlight the role of MS pathways. For the latter, the WT analysis demonstrates that the EXAFS spectrum is dominated by MS along the CO ligands and we explain into the insensitivity of the spectrum to the Re-X scattering pathway.

\section{THEORY}

The EXAFS signal, $\chi(k)$, is usually transformed into $R$ space using a FT, yielding a pseudo-radial distribution:

$$
\chi(R)=\int_{0}^{\infty} \chi(k) e^{i k R} d k
$$

Alternatively the $\mathrm{WT}^{22}$ consists in going beyond this $1 \mathrm{D}$ picture, yielding a 2D-correlation plot which deconvolutes the scattering pathways in both $R$ - and $k$-space, providing additional information about the different contributions to the
EXAFS spectrum. A WT is expressed as

$$
W_{f}^{\psi}\left(a, k^{\prime}\right)=\frac{1}{\sqrt{a}} \int_{-\infty}^{\infty} \chi(k) \psi^{*}\left(\frac{k-k^{\prime}}{a}\right) d k,
$$

where the scalar product of the EXAFS signal and the complex conjugate of the wavelet $\left(\psi^{*}\right)$ is calculated as a function of $a$ and $k^{\prime} . a$ is the scaling function, connected to $R$-space through the relation $a=\eta / 2 R$ and $k^{\prime}$ corresponds to the translation of the original wavelet as a function of the $k$-vector. In this work we have used the Morlet wavelet expressed as

$$
\psi(k)=\frac{1}{\sqrt{2 \pi} \sigma} e^{i \eta k} e^{-k^{2} / 2 \sigma^{2}},
$$

with the condition that

$$
\int_{-\infty}^{\infty} \psi(k) d k=0 .
$$

It is important to note that both $\eta$ and $\sigma$ (Eq. (6)), which correspond to the frequency of the sine or cosine functions and the width of the Gaussian, respectively, ${ }^{18}$ play an important role in the analysis. The resolution of the wavelets is analogous to a Gaussian normal distribution and a WT distributes the information over $k-R$ cells, often referred to as Heisenberg boxes. For a Morlet wavelet the $k-R$ cells are expressed as

$$
\left[k \pm \frac{n \sigma}{2^{1 / 2} R}\right] \times\left[R \pm \frac{R}{2^{1 / 2} n \sigma}\right]
$$

Therefore the $k-R$ window will be narrow in $k$ space for large $R$ and wide for small $R$. In $R$-space the resolution decreases by increasing $R$. However, it is important to note that this resolution can be optimised for a particular purpose by adjusting the wavelet parameters $\eta$ and $\sigma$, as demonstrated below.

\section{A. Computational details}

All of the spectra presented in the following work are derived from experimental data, except those presented in Figs. 8 and 9. For these, the simulations, which concern the rhenium complex, were performed using the FEFF9 package ${ }^{23}$ based on the DFT(B3LYP) optimised geometry, which can be found in Ref. 24. In each case, the calculated EXAFS spectrum was simulated using a maximum scattering path length of $6.0 \AA$ with up to 4 scattering legs (1 leg would be the scattering from the absorbing atom to a neighbour and back again). The amplitude reduction factor $\left(S_{0}^{2}\right)$ was 0.9 and the global Debye-Waller factor was $0.004 .^{25}$ Details of the best fit EXAFS spectrum, presented in Fig. 6, can be found in Ref. 25.

To support the analysis of the iron hexacyanide spectra the important scattering paths, reported in Table I, were calculated, also with the FEFF9 package $^{23}$ using the structure refined by Hayakawa et al. ${ }^{26}$

\section{RESULTS}

To investigate the potential of the WT analysis for molecular systems we apply this approach to study two transition metal complexes, namely iron hexacyanide, in both its ferrous and ferric forms and a rhenium diimine complex, 
TABLE I. The details of the four most important scattering paths for $\left[\mathrm{Fe}(\mathrm{CN})_{6}\right]^{4-}$. In brackets are the value for $\left[\mathrm{Fe}(\mathrm{CN})_{6}\right]^{3-}$ if different from $\left[\mathrm{Fe}(\mathrm{CN})_{6}\right]^{4-} . \mathrm{C}_{c w}$ is the percentage importance of the scattering path relative to the first single scattering path $(\mathrm{Fe}-\mathrm{C})$ and NLEG denotes the number of scattering legs, i.e., 1 is single scattering and $\mathrm{n}>1$ is multiple scattering. $R$ is the path distance and $\mathrm{N}_{\gamma}$ is the degeneracy.

\begin{tabular}{lccrc}
\hline \hline Path & $R(\AA)$ & $\mathrm{C}_{c w}$ & $\mathrm{~N}_{\gamma}$ & NLEG \\
\hline $1(\mathrm{Fe}-\mathrm{C})$ & $1.90(1.93)$ & 100.0 & 6 & 1 \\
$2(\mathrm{Fe}-\mathrm{N})$ & $3.08(3.11)$ & $32.6(33.0)$ & 6 & 1 \\
$3(\mathrm{Fe}-\mathrm{C}-\mathrm{N})$ & $3.08(3.11)$ & $161.6(163.9)$ & 12 & 2 \\
$4(\mathrm{Fe}-\mathrm{C}-\mathrm{N}-\mathrm{C})$ & $3.08(3.11)$ & $201.2(203.3)$ & 6 & 3 \\
\hline \hline
\end{tabular}

$\left[\operatorname{ReX}(\mathrm{CO})_{3}(\mathrm{bpy})\right],{ }^{24,25}$ where $\mathrm{X}=\mathrm{Br}, \mathrm{Cl}$ or Etpy. The structures of both complexes are shown in Fig. 1.

\section{A. Iron hexacyanide}

The nature of chemical bonds between transition metals and their ligands plays a fundamental role in coordination chemistry. For this reason, ferrous (Fe(II)) and ferric (Fe(III)) hexacyanides, $\left[\mathrm{Fe}(\mathrm{CN})_{6}\right]^{4-}$ and $\left[\mathrm{Fe}(\mathrm{CN})_{6}\right]^{3-}$, have been widely studied using a variety of theoretical ${ }^{27-29}$ and experimental techniques. ${ }^{30-32}$ Bianconi et al ${ }^{33}$ performed one of the first XAS characterisations of these complexes at the Fe K-edge and reported Fe-C distances of $1.90 \AA$ and $\mathrm{C}-\mathrm{N}$ distances of $1.19 \AA$ for the ferrous complex. Upon oxidation, forming the ferric complex a small expansion of the Fe-C distance by $0.03 \AA$ is observed while the $\mathrm{C}-\mathrm{N}$ distance remains constant. A later study by Hayakawa et al. ${ }^{26}$ demonstrated that the resonances in the XANES and EXAFS regions of these spectra are dominated by MS pathways along the linear bonds, known as the focusing effect. ${ }^{34}$ Given its high symmetry and well studied EXAFS spectrum, iron hexacyanide represents an ideal system to assess the performance of WT for molecules, which we now present.

The EXAFS spectra we obtained for both $\left[\mathrm{Fe}(\mathrm{CN})_{6}\right]^{4-}$ and $\left[\mathrm{Fe}(\mathrm{CN})_{6}\right]^{3-}$ dissolved in water are plotted in Fig. 2(a) with a $k^{2}$ weighting, and the corresponding FTs are shown in Fig. 2(b). The pseudo-radial distribution function shows, in accord with previous studies, two main peaks at 1.4 and $2.5 \AA$ which correspond to the $\mathrm{Fe}-\mathrm{C}$ and $\mathrm{Fe}-\mathrm{N}$ distances (not phase corrected), respectively. We also observe for the ferric case a very small shift of the $\mathrm{Fe}-\mathrm{C}$ peak to larger $R$ corresponding to the $0.03 \AA$ bond elongation in comparison to the ferrous

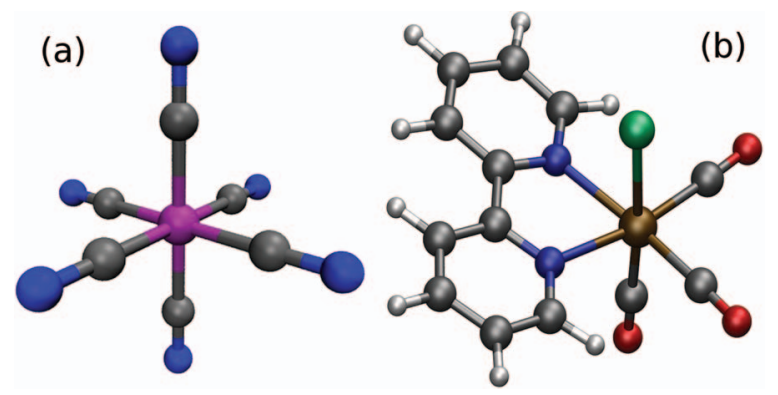

FIG. 1. Molecular structures of iron hexacyanide (a) and $\left[\operatorname{ReBr}(\mathrm{CO})_{3}(\mathrm{bpy})\right]$ (b). Colour code: $\mathrm{Fe}=$ magenta, $\mathrm{C}=$ grey, $\mathrm{N}=$ blue, $\mathrm{Br}=$ green, and Re $=$ brown.
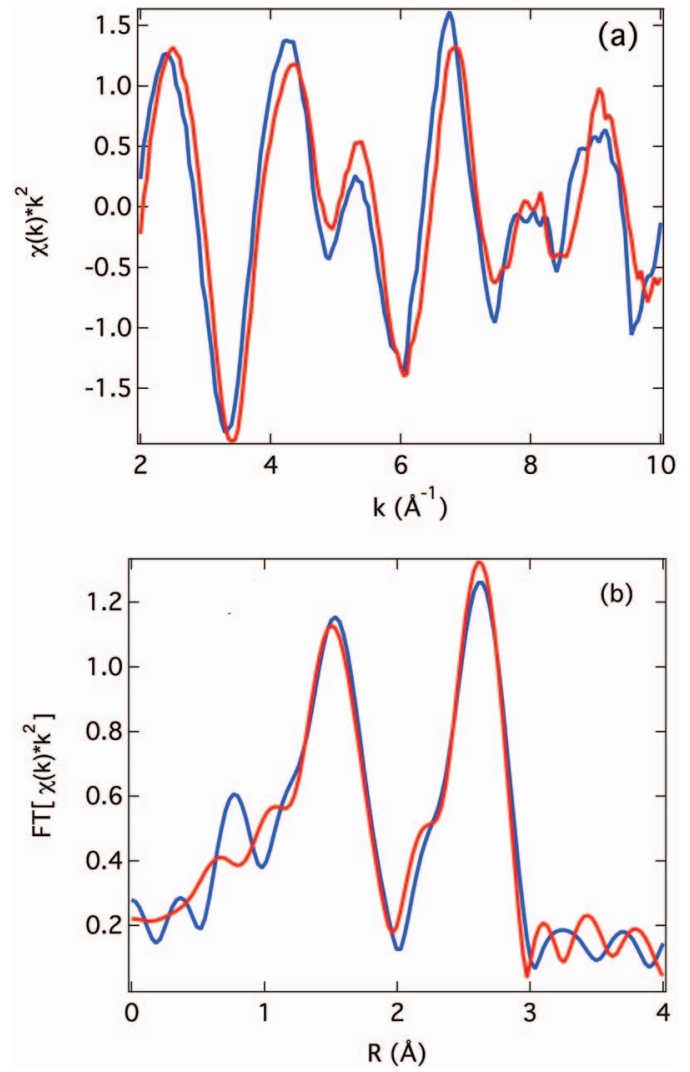

FIG. 2. (a) The Fe K-edge EXAFS spectrum of $\left[\mathrm{Fe}(\mathrm{CN})_{6}\right]^{4-}$ (red) and $\left[\mathrm{Fe}(\mathrm{CN})_{6}\right]^{3-}$ (blue) dissolved in water. Both spectra have been weighted by $k^{2}$. The $k$-space range shown corresponds to $\sim 7.13-7.44 \mathrm{keV}$. (b) The Fourier transform of (a) which yields the associated pseudo-radial distribution function.

complex. The corresponding shift is not clear in the Fe-N peak in agreement with Refs. 26 and 33.

The WT of the EXAFS spectra of Fig. 2(a) are shown in Figs. 3(a) and 3(b). In this first case, due to the small change in the bond length between these complexes we require a high resolution in $R$-space and have therefore used the wavelet parameters, $\eta=10.5$ and $\sigma=1.5$ (Eq. (8)). As a consequence, the resulting WTs are relatively featureless in $k$-space. We observe, as for the FT that the WT for the ferrous complex yields two main features in $R$-space centred around 1.4 and $2.5 \AA$ corresponding to the $\mathrm{Fe}-\mathrm{C}$ and $\mathrm{Fe}-\mathrm{N}$ distances (not phase corrected). This is also true for the ferric complex, which in

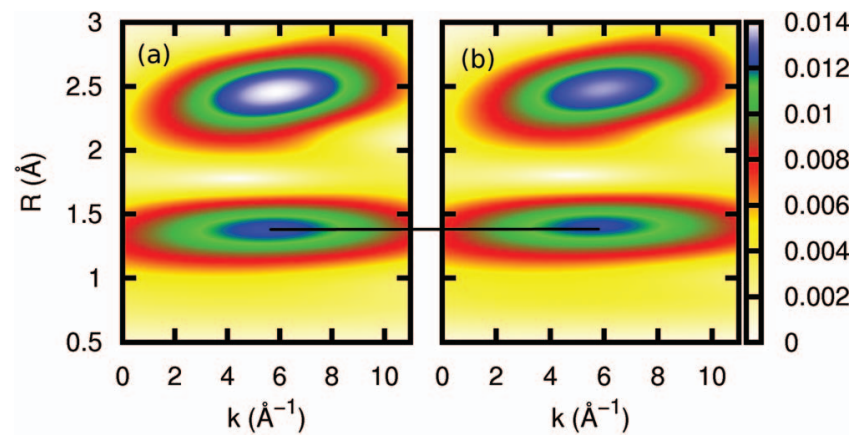

FIG. 3. The Wavelet transform of the experimental EXAFS spectra at the Fe $\mathrm{K}$-edge of (a) $\left[\mathrm{Fe}(\mathrm{CN})_{6}\right]^{4-}$ and (b) $\left[\mathrm{Fe}(\mathrm{CN})_{6}\right]^{3-}$. For this analysis we have used $\eta=10.5$ and $\sigma=1.5$. 


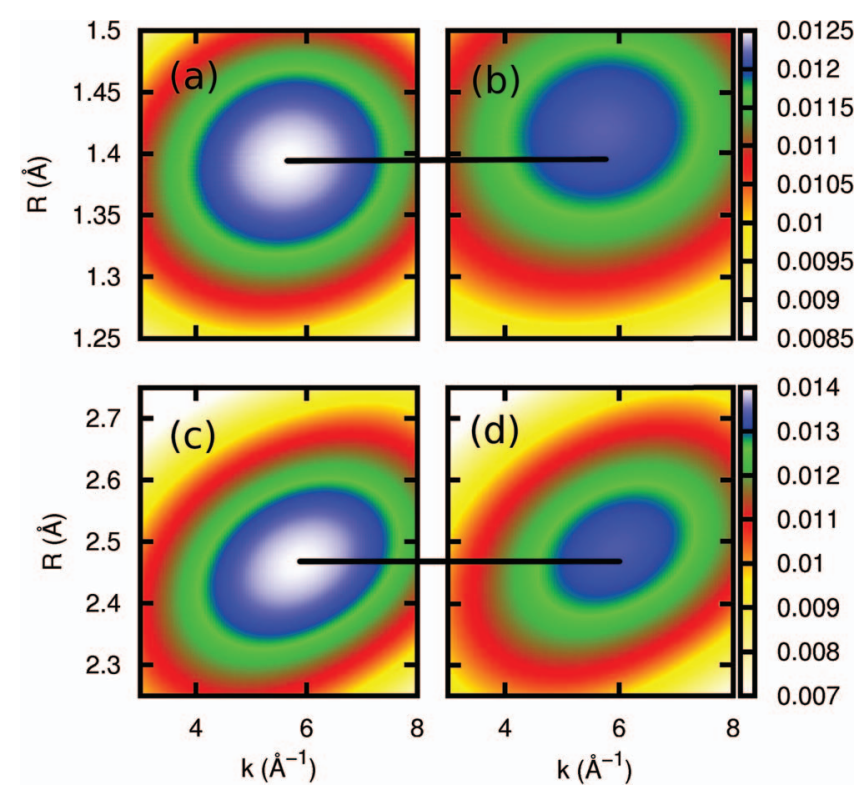

FIG. 4. A zoom of the two features shown in the Wavelet transforms of Fig. 3. (a) and (c) The ferrous complex; (b) and (d) the ferric complex.

addition exhibits the small shift to larger $R$ for the first peak, highlighted by the straight black line running between the plots and seen more clearly in the zoom of this feature shown in Figs. 4(a) and 4(b) for the ferrous and ferric complex, respectively. This offset, in agreement with the FT, corresponds to $\sim 0.03 \AA$. For the second feature (for which a zoom is shown in Figs. 4(c) and 4(d)) we observe a slight weakening of its intensity in the case of the ferric complex consistent with the FT. The broad nature of the feature means that it is harder to pinpoint the change in the $\mathrm{Fe}-\mathrm{N}$ distance, but in contrast to the FT there does appear to be a small shift.

Interestingly, this peak in both spectra exhibits a distinct chirped shape. Such a chirp can arise from either a nonlinear $k$-dependence of the backscatter phase ${ }^{18}$ in Eq. (3), or from two features overlapping in $k$-space which differ slightly in $R$. To assess this, Figs. 5(a) and 5(b) show the WT analysis of $\left[\mathrm{Fe}(\mathrm{CN})_{6}\right]^{4-}$ and $\left[\mathrm{Fe}(\mathrm{CN})_{6}\right]^{3-}$ for which the wavelet parameters are $\eta=7.5$ and $\sigma=0.5$. This decreases the resolution in $R$-space, but increases it in $k$-space permitting a more detailed description of the chirp. The WT analysis in this case shows

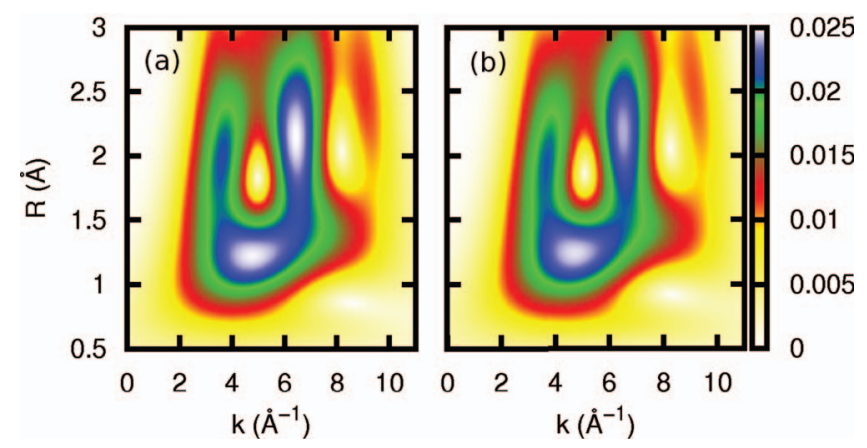

FIG. 5. The Wavelet transform of the experimental EXAFS spectra at the Fe K-edge of (a) $\left[\mathrm{Fe}(\mathrm{CN})_{6}\right]^{4-}$ and (b) $\left[\mathrm{Fe}(\mathrm{CN})_{6}\right]^{3-}$. For this analysis we have used $\eta=7.5$ and $\sigma=0.5$. distinct differences from the previous spectra shown in Fig. 3, and this is primarily observed in the 3 regions which appear in $k$-space $\left(4,6.5\right.$, and $\left.9 \AA^{-1}\right)$ at larger $R$.

Here, the spectrum is principally composed of the SS pathway $\mathrm{Fe}-\mathrm{N}$ and the MS pathways, $\mathrm{Fe}-\mathrm{C}-\mathrm{N}$ and $\mathrm{Fe}-\mathrm{C}-\mathrm{N}-$ $\mathrm{C}$, as shown by the percentage importance of the scattering paths, $\mathrm{C}_{c w}$, in Table I. The 3 peaks in the WT at larger $R$ arise from an interference between these pathways and the Fe-C scattering pathway. However, all three have the same length and therefore cannot explain the chirp reported in Fig. 3, which as a consequence must arise from a slight nonlinear $k$-dependence of the backscatter phase.

For the Fe-C feature, the reduced resolution in $R$-space means that we now observe a broader feature, which has merged with the one at larger $R$. The WT also shows a slight broadening of this feature to $R<1 \AA$ and between $k=2-$ $6 \AA^{-1}$. This arises from the resolution parameters (Eq. (8)). This is not present in the previous WT (Fig. 3) because of the higher resolution in $R$-space. However, in this second case the parameters means that the $k-R$ cell is broader in this region. Overall, this analysis demonstrates that the wavelet parameters used in this case provide insight into the scattering pathways which contribute to the spectrum, but do make it difficult to give a description of the structure as for the previous WT. This is especially true for the change in the $\mathrm{Fe}-\mathrm{C}$ bond length between the ferric and ferrous cases which is masked by the broad nature of the features. In addition, the peak of the Fe$\mathrm{C}$ feature appears to shift to smaller $R$, being centred around $1.25 \AA$, rather than $1.4 \AA$ as in the case of Fig. 3. This, as demonstrated by Funke et al. ${ }^{18}$ is a consequence of the resolution (the $\sigma$ and $\eta$ parameters) and it therefore highlights that a thorough understanding of their effect must be obtained in this type of analysis.

\section{B. Rhenium diimine}

In Sec. III A, we characterised the WT of the EXAFS spectra for the well studied iron hexacyanide complexes and demonstrated that, in addition to the information which can be extracted from the FT, i.e., the Fe-C and Fe-N distances, this method can yield additional details, such as the contribution of certain scattering pathways and the nonlinear $k$ dependence of the backscatter phase. We now extend this to a more complex system, namely $\left[\operatorname{ReX}(\mathrm{CO})_{3}(\mathrm{bpy})\right]$ where $\mathrm{X}=\mathrm{Br}, \mathrm{Cl}$, or Etpy, which we recently characterised by static and picosecond-XAS. ${ }^{25}$ For the present purposes, the low symmetry of the complex means that there are many overlapping (in $R$-space) scattering pathways and therefore this complex represents a significant challenge for EXAFS analysis. In addition, the aforementioned investigation reported that the $\mathrm{Re} \mathrm{L}_{3}$-edge spectrum is very similar when the $\mathrm{Br}$ ligand is exchanged for either $\mathrm{Cl}$ or Etpy, indicating that the spectrum is rather insensitive to the $\mathrm{Re}-\mathrm{X}$ scattering pathway. This is surprising given the size of the ligand $X$. Thereafter we analyse the experimental and best fit EXAFS spectrum of the $\mathrm{X}=\mathrm{Br}$ complex. Then, using the DFT optimised geometries, we perform a WT analysis on the calculated EXAFS spectra for all three complexes. 

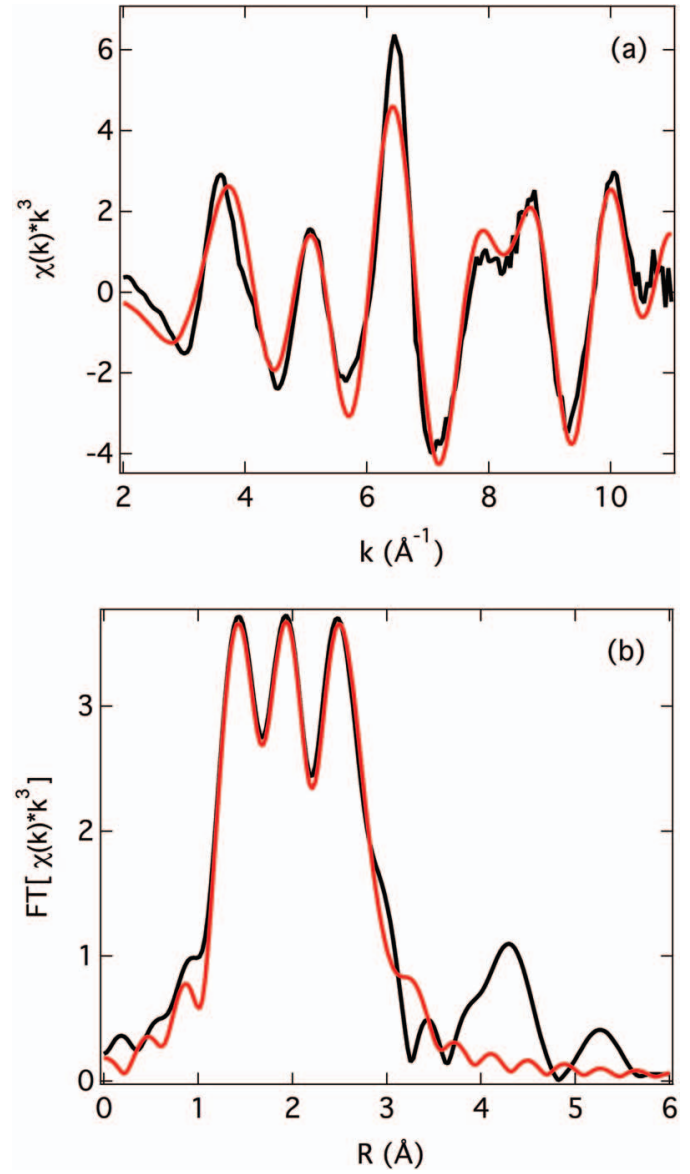

FIG. 6. (a) The Re $\mathrm{L}_{3}$-edge best fit (red) and experimental (black) EXAFS spectrum of $\left[\operatorname{ReBr}(\mathrm{CO})_{3}(\mathrm{bpy})\right]$ dissolved in acetonitrile. The $k$-space range shown corresponds to $\sim 10.55-11.2 \mathrm{keV}$. (b) The associated pseudoradial distribution function. The feature in the experimental spectrum at larger $R, 4.0 \AA$, which is not reproduced in the best fit spectrum is because scattering pathways of this length were not included as this led to too many fitting parameters and a violation of the Nyquist criterion. ${ }^{17}$

Fig. 6(a) shows the $\operatorname{Re} \mathrm{L}_{3}$-edge best fit (red) and experimental (black) EXAFS spectrum of $\left[\operatorname{ReBr}(\mathrm{CO})_{3}\right.$ (bpy)] dissolved in acetonitrile, previously presented in Ref. 25. The fit was performed with the IFEFFIT package ${ }^{14}$ and includes the $\mathrm{Re}-\mathrm{C}_{\mathrm{co}}, \mathrm{Re}-\mathrm{N}, \mathrm{Re}-\mathrm{Br}$, and Re-O scattering pathways along with two non-structural parameters; the amplitude reduction factor and the ionisation potential. The overall agreement between the fit and the experimental spectrum is good and the corresponding FT (Fig. 6(b)) shows three distinct peaks at 1.5, 1.9 , and $2.5 \AA$. There is also a small feature in the experimental spectrum at larger $R, 4.0 \AA$, which is not reproduced in the best fit spectrum because scattering pathways of this length were not included as this led to too many fitting parameters and a violation of the Nyquist criterion. ${ }^{17}$

Figs. 7(a) and 7(b) show the WT of the XAS best fit and experimental $\operatorname{Re} \mathrm{L}_{3}$-edge EXAFS spectrum of $\left[\operatorname{ReBr}(\mathrm{CO})_{3}(\mathrm{bpy})\right]$. Due to signal-to-noise $(\mathrm{S} / \mathrm{N})$ considerations the experimental spectrum was recorded up to $11.2 \mathrm{keV}$, corresponding to $11 \AA^{-1}$ and therefore the XAS best fit spectrum is also shown for this range. The overall agreement between the two spectra is good and both exhibit three principal regions; a peak at $\sim 1.5 \AA$ which traverses a large portion of
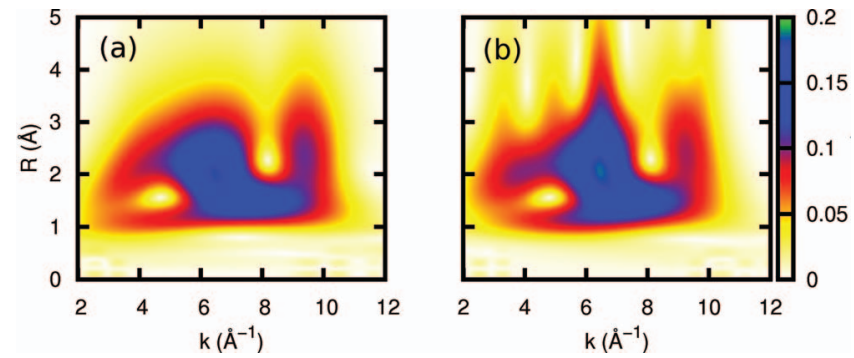

FIG. 7. The Wavelet transform of the XAS best fit (a) and experimental (b) EXAFS spectrum of the $\operatorname{Re} \mathrm{L}_{3}$-edge of $\left[\operatorname{ReBr}(\mathrm{CO})_{3}(\mathrm{bpy})\right]$. For this analysis we have used $\eta=7.5$ and $\sigma=0.5$.

$k$-space, and two peaks at $\sim 2.5 \AA$, which are centred at 6 and $9.5 \AA^{-1}$, respectively.

To understand the origin of these features, Fig. 8 shows the WT of 4 calculated EXAFS spectra, using the XAS best fit geometry and gradually increasing the number of scattering pathways. It is important to note that in this case we have calculated the spectrum up to $15 \AA^{-1}$. This region is not included in Fig. 6 because it could not be recorded with a sufficient $\mathrm{S} / \mathrm{N}$; however, we present it here to highlight the role of scattering pathways at higher $k$.

Fig. 8(a) includes only the Re- $\mathrm{C}_{\mathrm{co}}$ scattering pathways, and the resulting WT exhibits a broad peak at $R \sim 1.5 \AA$ over a $k$ range of $3-13 \AA^{-1}$, corresponding to the Re-C distance (not phase corrected) and the first peak in the FT (Fig. 6(b)). Upon inclusion of the carbonyl oxygens (Fig. 8(b)), a significant change is observed. Additional features appear at $\sim 2.5 \AA$ in $R$-space corresponding to the $\mathrm{Re}-\mathrm{O}$ distance, the third peak in the FT (Fig. 6(b)). In $k$-space 4 distinct regions are formed between 4 and $13 \AA^{-1}$ for which the strongest is around $10 \AA^{-1}$. In a similar manner to previously reported for iron hexacyanide these peaks arise from the interference between the Re-C scattering pathway and the scattering pathways which contribute to the higher region of $R$-space, namely Re$\mathrm{O}$ and Re-C-O as shown in Table II. This WT also exhibits the
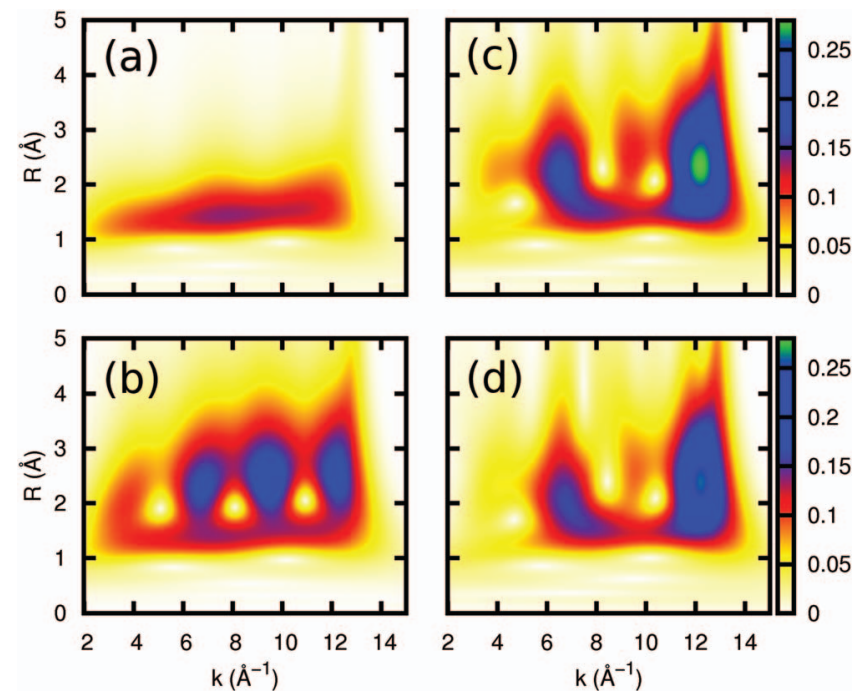

FIG. 8. The Wavelet transform of calculated EXAFS signals for (a) $\mathrm{ReC}_{3}$, (b) $\operatorname{Re}(\mathrm{CO})_{3}$, (c) $\operatorname{ReBr}(\mathrm{CO})_{3}$, and (d) $\operatorname{ReBr}(\mathrm{CO})_{3}$ bpy. For this analysis we have used $\eta=7.5$ and $\sigma=0.5$. 
TABLE II. The details of the 6 most important scattering paths for $\left[\operatorname{ReBr}(\mathrm{CO})_{3}\right.$ bpy]. $\mathrm{C}_{c w}$ is the percentage importance of the scattering path relative to the first single scattering path $\left(\mathrm{Re}-\mathrm{C}_{C O}\right)$ and NLEG denotes the number of scattering legs, i.e., 1 is single scattering and $n>1$ is multiple scattering. $R$ is the path distance and $\mathrm{N}_{\gamma}$ is the degeneracy.

\begin{tabular}{lcccc}
\hline \hline Path & $R(\AA)$ & $\mathrm{C}_{c w}$ & $\mathrm{~N}_{\gamma}$ & NLEG \\
\hline $1\left(\operatorname{Re}-\mathrm{C}_{C O}\right)$ & 1.92 & 100.0 & 3 & 1 \\
$2(\operatorname{Re}-\mathrm{N})$ & 2.22 & 48.1 & 2 & 1 \\
$3(\operatorname{Re}-\mathrm{Br})$ & 2.67 & 25.48 & 1 & 1 \\
$4\left(\operatorname{Re}-\mathrm{C}_{b p y}\right)$ & 3.08 & 18.68 & 2 & 1 \\
$5(\operatorname{Re}-\mathrm{O})$ & 3.08 & 29.86 & 3 & 1 \\
$6(\operatorname{Re}-\mathrm{C}-\mathrm{O})$ & 3.08 & 116.50 & 6 & 2 \\
\hline \hline
\end{tabular}

chirped profile discussed in Sec. III A, and again this arises from a nonlinear $k$-dependence of the backscattering phase function. In this case it is larger than for iron hexacyanide, as one would expect for a heavier absorbing atom, Re.

When the Br scattering pathway is included (Fig. 8(c)), the most pronounced changes are observed at high $k$-values, as expected for a heavy scatterer. In particular the feature observed at $k \sim 9.5 \AA^{-1}$ is significantly reduced and the feature at $k \sim 12 \AA^{-1}$ becomes broader and stronger. However, importantly the latter $\left(k \sim 12 \AA^{-1}\right)$ is not included in the experimental spectrum (Fig. 6(a)) because it is well $(\sim 500 \mathrm{eV})$ above the edge and achieving a good $\mathrm{S} / \mathrm{N}$ in this region is a significant challenge. This highlights that when fitting the EXAFS spectrum, its dependence on the $\mathrm{Re}-\mathrm{Br}$ bond length is likely to be weak and therefore extracting an accurate bond length is expected to be difficult. The $k$-space requirements to accurately fit bond lengths of heavy scatterers has been previously discussed in detail, especially for bimetallic complexes embedded in biological media. ${ }^{13}$ In particular Blackburn et al $^{35}$ have shown that to obtain an accurate description of the $\mathrm{Cu}-\mathrm{Cu}$ distance in cytochrome oxidase, the spectrum needs to be fitted up to $16 \AA^{-1}$, while a similar conclusion was drawn by Hedman et al. for the Fe-Fe distance in binuclear iron complexes. ${ }^{36}$

Fig. 8(d) shows the WT transform of the calculated EXAFS spectrum for the entire molecule. The simulated spectrum now, unlike the best fit spectrum (Fig. 7(a)), includes, to some extent the features at larger $R\left(k \sim 7 \AA^{-1}\right)$. However, there are deviations from the WT of the experimental EXAFS spectrum (Fig. 7(b)), which is more easily compared using Fig. 9(c), which plots the WT analysis in Fig. 8(d), limited to the same $k$-space range as Fig. 7. These differences indicate that the structure is not in perfect agreement with the experiment. This is not unsurprising considering the complex nature of the molecule and the limitations of the fit associated with the Nyquist criterion as described above. However, using the previous WT analysis we are able to postulate that these deviations are likely to correspond to the $\mathrm{Re}-\mathrm{Br}$ and $\mathrm{Re}-$ $\mathrm{C}-\mathrm{O}$ scattering pathways because these regions $(k \sim 3$ and $10 \AA^{-1}$ ) of the WT in Fig. 8(d) exhibit the poorest agreement. This demonstrates the potential scope provided by the WT analysis, where the deviations between theoretical and experimental EXAFS spectra can be associated with a particular scattering pathway and therefore systematically improved.
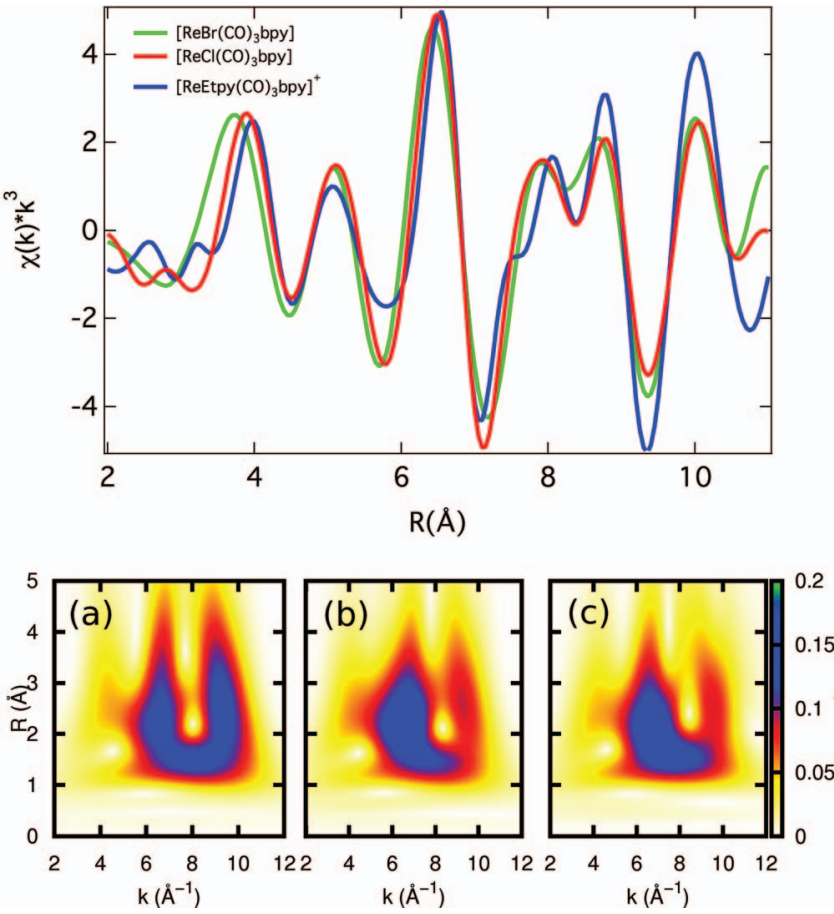

FIG. 9. (Upper panel) The calculated EXAFS spectra using the DFT optimised geometries ${ }^{25}$ of $\left[\operatorname{ReEtpy}(\mathrm{CO})_{3}(\mathrm{bpy})\right]^{+}$(blue), $\left[\operatorname{ReCl}(\mathrm{CO})_{3}(\right.$ bpy $\left.)\right]$ (red), and $\left[\operatorname{ReBr}(\mathrm{CO})_{3}(\mathrm{bpy})\right]$ (green). (Lower panel) The Wavelet transform of calculated EXAFS signals for (a) $\left[\operatorname{ReEtpy}(\mathrm{CO})_{3}(\mathrm{bpy})\right]$, (b) $\left[\operatorname{ReCl}(\mathrm{CO})_{3}(\mathrm{bpy})\right]$, and (c) $\left[\operatorname{ReBr}(\mathrm{CO})_{3}(\mathrm{bpy})\right]$. For this analysis we have used $\eta=7.5$ and $\sigma=0.5$.

Finally, as previously mentioned, Ref. 25 showed that the $\mathrm{Re} \mathrm{L}_{3}$-edge spectrum was rather insensitive to exchanges of the $\mathrm{Br}$ for either $\mathrm{Cl}$ or Etpy. The upper panel of Fig. 9 shows the EXAFS spectra for $\left[\operatorname{ReEtpy}(\mathrm{CO})_{3}(\mathrm{bpy})\right]^{+}$(blue), $\left[\operatorname{ReCl}(\mathrm{CO})_{3}(\right.$ bpy $\left.)\right]\left(\right.$ red), and $\left[\operatorname{ReBr}(\mathrm{CO})_{3}(\right.$ bpy $\left.)\right]$ (green) calculated using the DFT optimised geometries. ${ }^{24}$ The WT analysis for each of these spectra is shown in the Figs. 9(a)9(c) (lower panel). In this case, our previous analysis makes it possible to identify the complex using the differences between the WT. Most notably the feature at $k=9.5 \AA^{-1}$ is much stronger in Fig. 9(a). It was demonstrated in Fig. 8 that this feature is significantly damped by the presence of the heavy scatterer and therefore its intensity is indicative of the $\mathrm{X}=$ Etpy complex, i.e., when a heavy scatterer is absent. This can also be applied to Fig. 9(b) for which this peak is slightly stronger than in Fig. 9(c), indicating a smaller scattering, consistent with $\mathrm{X}=\mathrm{Cl}$. Importantly, this qualitative analysis would not be directly possible using the traditional FT, because the many overlapping scattering pathways and the limitations of $k$-space associated to the $\mathrm{S} / \mathrm{N}$ mean that the pseudo-radial distribution does not strongly reflect features of the Re-X scattering.

\section{CONCLUSION}

In this paper we have presented for the first time, a WT analysis of the EXAFS spectra of molecules. We have applied it to the study of two transition metal complexes, namely iron hexacyanide in both its ferric and ferrous form, and a rhenium diimine complex, $\left[\operatorname{ReX}(\mathrm{CO})_{3}(\mathrm{bpy})\right], \mathrm{X}=\mathrm{Etpy}, \mathrm{Cl}$, or $\mathrm{Br}$. Our 
results demonstrate that such an analysis provides additional insight into the composition of the EXAFS spectrum in terms of the identity of the scattering atom and the importance of multiple scattering pathways. For the rhenium diimine complex, we have been able to obtain direct insight into the limits of the analysis in terms of the Re-X bond length. For iron hexacyanide, we have also shown the important role of the wavelet parameters, which controls the resolution of the analysis. This is defined by the $k-R$ cells and can be the limiting factor of such an analysis. However, these can be tailored to the particular requirement of a system, and we stress that in comparison to the FT method, although on occasions, primarily for simple and symmetric systems, the WT approach may not deliver additional information about the EXAFS spectrum, it will never deliver less.

Importantly, this method should be extended into the time-domain XAS, ${ }^{37}$ for which changes in the WT could be directly associated with changes of the structure, offering many potentially exciting opportunities. This is likely to be particularly pertinent in the near future because of the recent advances in the methodologies of time-resolved XAS, such as high-repetition rate pump lasers, ${ }^{38}$ which has led to a significant improvement in $\mathrm{S} / \mathrm{N}$. In addition coupled with the potential advances bought about by $\mathrm{X}$-ray Free-electron lasers (X-FELs) it should be possible to obtain high signal to noise transient EXAFS signals of molecules in solution. In such cases, the additional degree of freedom (time) can make analysis of the EXAFS spectrum using traditional methods challenging because one must include not only the structural changes from the ground state, but also the photolysis yield. ${ }^{39}$ This is sometimes difficult to directly derive from the experiment and therefore is an adjustable parameter in the fit. ${ }^{40}$

Finally this work represents a starting point to the future development of this approach. In particular it is important to implement this in conjunction with a fitting procedure, for which one could expect that this approach will offer a more detailed and unambiguous description of the molecular structure from the EXAFS spectrum, especially for complex molecules.

\section{ACKNOWLEDGMENTS}

This work was funded by the Swiss National Science Foundation (NSF) through the NCCR MUST "Molecular ultrafast science and technology" and Contract No. 200021137717.
${ }^{1}$ G. Bunker, Introduction to XAFS: A Practical Guide to X-ray Absorption Fine Structure (Cambridge University Press, 2010).

${ }^{2}$ J. Stöhr, NEXAFS Spectroscopy, Springer Series in Surface Sciences Vol. 25 (Springer, 1996).

${ }^{3}$ P. Lee and G. Beni, Phys. Rev. B 15, 2862 (1977).

${ }^{4}$ F. de Groot, Chem. Rev. 101, 1779 (2001).

${ }^{5}$ P. Lee, P. Citrin, P. Eisenberger, and B. Kincaid, Rev. Mod. Phys. 53, 769 (1981).

${ }^{6}$ J. Rehr and R. Albers, Phys. Rev. B 41, 8139 (1990).

${ }^{7}$ J. Rehr, Rev. Mod. Phys. 72, 621 (2000).

${ }^{8}$ C. Natoli, M. Benfatto, C. Brouder, M. Ruiz Lopez, and D. Foulis, Phys. Rev. B 42, 1944 (1990).

${ }^{9}$ A. Filipponi, A. Di Cicco, and C. Natoli, Phys. Rev. B 52, 15122 (1995).

${ }^{10}$ Formally, MS approaches are valid both below and above the edge, and the only limitation for the potential is that the atomic cells are non-overlapping.

${ }^{11}$ D. Sayers, E. Stern, and F. Lytle, Phys. Rev. Lett. 27, 1204 (1971).

${ }^{12}$ The distances found in the Fourier transformation are about 0.2-05 shorter than the actual distance due to the energy dependence of the phase factors.

${ }^{13}$ P. J. Riggs-Gelasco, T. L. Stemmler, and J. E. Penner-Hahn, Coord. Chem. Rev. 144, 245 (1995).

${ }^{14}$ M. Newville, J. Synchrotron Radiat. 8, 322 (2001).

${ }^{15}$ D. Koningsberger, B. Mojet, G. Van Dorssen, and D. Ramaker, Top. Catal. 10, 143 (2000).

${ }^{16}$ M. Stearns, Phys. Rev. B 25, 2382 (1982).

${ }^{17}$ E. Stern, Phys. Rev. B 48, 9825 (1993).

${ }^{18}$ H. Funke, A. Scheinost, and M. Chukalina, Phys. Rev. B 71, 094110 (2005).

${ }^{19}$ H. Funke, M. Chukalina, and A. Scheinost, J. Synchrotron Radiat. 14, 426432 (2007).

${ }^{20}$ M. Munoz, F. Farges, and P. Argoul, Phys. Scr. T115, 221 (2005).

${ }^{21}$ R. O. Savinelli and S. L. Scott, Phys. Chem. Chem. Phys. 12, 5660 (2010).

${ }^{22}$ G. Kaiser, A Friendly Guide to Wavelets (Birkhäuser, Boston, 1994).

${ }^{23}$ J. Rehr et al., C. R. Phys. 10, 548 (2009).

${ }^{24}$ A. El Nahhas et al., J. Phys. Chem. A 114, 6361 (2010).

${ }^{25} \mathrm{~A}$. El Nahhas, "Time-resolved optical and x-ray spectroscopy of rhenium based molecular complexes," Ph.D. dissertation (EPFL, 2010).

${ }^{26}$ K. Hayakawa et al., J. Am. Chem. Soc. 126, 15618 (2004).

${ }^{27}$ H. Bolvin, J. Phys. Chem. A 102, 7525 (1998).

${ }^{28}$ S. DeBeer-George, T. Petrenko, and F. Neese, J. Phys. Chem. A 112, 12936 (2008).

${ }^{29}$ N. Lee, T. Petrenko, U. Bergmann, F. Neese, and S. DeBeer, J. Am. Chem. Soc. 132, 9715 (2010).

${ }^{30}$ R. Hocking et al., J. Am. Chem. Soc. 128, 10442 (2006).

${ }^{31}$ M. Obashi, Jpn. J. Appl. Phys. 17, 563 (1978).

${ }^{32}$ J. J. Alexander and H. B. Gray, J. Am. Chem. Soc. 90, 4260 (1968).

${ }^{33}$ A. Bianconi, M. Dell'Ariccia, P. J. Durham, and J. B. Pendry, Phys. Rev. B 26, 6502 (1982).

${ }^{34}$ S. I. Zabinsky, J. J. Rehr, A. Ankudinov, and R. C. Albers, Phys. Rev. B 52, 2995 (1995).

${ }^{35}$ N. J. Blackburn, M. E. Barr, W. H. Woodruff, J. van der Ooost, and S. de Vries, Biochemistry 33, 10401 (1994).

${ }^{36}$ B. Hedman, M. S. Co, W. H. Armstrong, K. O. Hodgson, and S. J. Lippard, Inorg. Chem. 25, 3708 (1986).

${ }^{37}$ C. Bressler and M. Chergui, Ann. Rev. Phys. Chem. 61, 263 (2010).

${ }^{38}$ F. Lima et al., Rev. Sci. Instrum. 82, 063111 (2011).

${ }^{39}$ C. Bressler, R. Abela, and M. Chergui, Z. Kristallogr. 223, 308 (2008).

${ }^{40}$ W. Gawelda et al., J. Chem. Phys. 130, 124520 (2009). 